\title{
Properties of Porous TiNbZr Shape Memory Alloy Fabricated by Mechanical Alloying and Hot Isostatic Pressing
}

\author{
L.W. Ma, C.Y. Chung, Y.X. Tong, and Y.F. Zheng
}

(Submitted May 15, 2010; in revised form February 16, 2011)

\begin{abstract}
In the past decades, systematic researches have been focused on studying Ti-Nb-based SMAs by adding ternary elements, such as Mo, Sn, $\mathrm{Zr}$, etc. However, only arc melting or induction melting methods, with subsequent hot or cold rolling, were used to fabricate these Ni-free SMAs. There is no work related to powder metallurgy and porous structures. This study focuses on the fabrication and characterization of porous Ti-22Nb-6Zr (at.\%) shape memory alloys produced using elemental powders by means of mechanical alloying and hot isostatic pressing. It is found that the porous $\mathrm{Ti}-22 \mathrm{Nb}-6 \mathrm{Zr}$ alloys prepared by the HIP process exhibit a homogenous pore distribution with spherical pores, while the pores have irregular shape in the specimen prepared by conventional sintering. X-ray diffraction analysis showed that the solid solution-treated Ti-22Nb-6Zr alloy consists of both $\beta$ phase and $\alpha^{\prime \prime}$ martensite phase. Morphologies of martensite were observed. Finally, the porous Ti-22Nb-6Zr SMAs produced by both MA and HIP exhibit good mechanical properties, such as superior superelasticity, with maximum recoverable strain of $\sim 3 \%$ and high compressive strength.
\end{abstract}

Keywords hot isostatic pressing, mechanical alloying, shape memory alloy

\section{Introduction}

Porous materials have been recently recognized as bone substitutes for a wide range of biomedical applications, involving high strength, a relatively low stiffness (attractive for minimizing stress-shielding effect) as well as the porous structure which permits bones tissue ingrowths, body fluid transmission and firm fixation of prosthesis, etc. Porous nickel-titanium (NiTi) shape memory alloy (SMA) is one of the most popular implanted materials that has attracted considerable interest owing to its shape memory effect (SME), super-elasticity (SE), and superior corrosion resistance (Ref 1). Also, the mechanical properties, such as Young's modulus, of these SMAs can be easily adjusted to match those of replaced bones by obtaining different porosities and pore sizes through controlling the synthesis conditions (Ref 2).

This article is an invited paper selected from presentations at Shape Memory and Superelastic Technologies 2010, held May 16-20, 2010, in Pacific Grove, California, and has been expanded from the original presentation.

L.W. Ma and C.Y. Chung, Department of Physics and Materials Science, City University of Hong Kong, 83 Tat Chee Avenue, Kowloon Tong, Hong Kong; and Y.X. Tong and Y.F. Zheng, Center for Biomedical Materials and Engineering, Harbin Engineering University, Harbin 150001, China. Contact e-mail: appchung@cityu. edu.hk.
However, it has been reported that the $\mathrm{Ni}$ ions released from these alloys can cause detrimental effect to humans, particularly for $\mathrm{Ni}$ hypersensitive patients resulting with strong allergic reactions (Ref 3 ). In order to overcome such problem, absolutely safe Ni-free Ti-based shape memory alloys (SMAs) are becoming increasingly used because they are non-toxic and bio-compatible (Ref 4).

It has been first reported by Baker (Ref 5 ) that a cold-rolled Ti-35 wt.\% Niobium ( $\mathrm{Nb}$ ) binary alloy exhibits shape memory effect. Miyazaki and co-workers (Ref 6) have performed a series of researches on studying the mechanical properties and morphologies of Ti-Nb-based alloys, fabricated by an arc melting method. Stable shape memory behavior and superelasticity were observed in Ti-(22-25)Nb (at.\%) and Ti- $(25.5-27) \mathrm{Nb}$ (at.\%) alloys, respectively. They concluded that solution-treated $\mathrm{Ti}-(25-27) \mathrm{Nb}$ (at.\%) gives the maximum recovered strain of $3 \%$. More recently, detailed investigations have been conducted on studying the shape memory effect of Ti-Nb-based alloys by adding ternary elements, including Molybdenum (Mo), Palladium (Pd), Tin (Sn), Zirconium (Zr), etc. Ping et al. (Ref 7) investigated the microstructural evolution of an arc-melted Ti-30Nb-3Pd (at.\%) shape memory alloys using TEM examination. Plated-shape martensitic $\omega, \alpha^{\prime \prime}$ martensite, retained $\beta$, and fine-particle $\omega$ phases were found. However, none of these studies have been performed using the traditional fabrication processes, such as powder metallurgy (PM) or mechanical alloying (MA).

It has been well documented in the literature that $\beta$ type Ti-based SMAs exhibit two martensitic transformations from the $\beta$-phase (disordered cubic) to either $\alpha^{\prime}$-phase (hexagonal) or $\alpha^{\prime \prime}$-phase (orthorhombic) by rapid quenching (Ref 4, 6). The shape memory effect of these Ti-based alloys is due to a reversible transformation between the martensitic $\alpha^{\prime \prime}$ - and the austenitic $\beta$-phases. It was also reported that the transformation 
temperature from the $\beta$-phase to the $\alpha^{\prime \prime}$-phase can be controlled by adjusting the amount of alloying elements. In order to achieve the required compositional control, these types of alloys are typically fabricated using an arc or induction melting followed by either hot or cold rolling to final dimensions. However, arc melting requires multiple re-melts to ensure homogeneity, while induction melting has the drawback of both crucible and oxygen contamination (Ref 8). Accordingly, nearnet-shape PM processing of Ti-based shape memory alloys has attracted considerable interest. It was reported that porous NiTi SMAs can been synthesized using different PM methods, including conventional sintering (CS) (Ref 9), self-propagating high-temperature synthesis (SHS) (Ref 10), spark plasma sintering (Ref 11), etc. However, such methods have resulted in substantial porosities and problems with dimensional control. On the other hand, sintering a mixture of elemental or prealloyed powders and space-holder particles, including $\mathrm{NaF}$ (Ref 12) and $\mathrm{NH}_{4} \mathrm{HCO}_{3}$ (Ref 13), in conjunction with HIP were also reported. Well-defined pore size, shape, and volume fraction were produced by eliminating the space-holder particles during or after densification. Despite a number of PM methods described, hot isostatic pressing (HIP) is one of the most promising techniques for SMAs production from elemental powders (Ref 14). The desired porosity and pore size of the SMA can be controlled by adjusting both pressure and temperature during the HIP to facilitate the formation of better near-net-shape components. The objective of this study is to develop Ni-free Ti-based shape memory alloy, Ti-22Nb-6Zr (at.\%), by means of the PM and the HIP techniques. Materials were examined at different stages of treatments including conventional sintering (CS), HIP as well as subsequent solid solution treated. Both martensitic phase and its morphology were found. Porosity was also measured to study the effect of HIP on superelasticity.

\section{Materials and Methods}

In this study, elemental metal powders of Ti with $>99 \%$ of purity and $<75 \mu \mathrm{m}$ of particle size (Sinopharm Chemical Reagent Co. Ltd., Beijing, China), $\mathrm{Nb}$ with $>99 \%$ of purity and $<75 \mu \mathrm{m}$ of particle size and $\mathrm{Zr}$ with $>99 \%$ of purity and $<75 \mu \mathrm{m}$ of particle size (Shanghai Richjoint Chemical Reagent Co. Ltd., Beijing, China) were used. These powder mixtures were ball-milled, to prepare Ti-22Nb-6Zr (at.\%) alloy (which will be referred to hereinafter as TiNbZr), using a planetary type GM-1F high-energy ball-milling machine. The ball-milling parameters are shown in Table 1.

The ball-milled powder mixtures were cold pressed into a number of green compacts $(\sim 15 \mathrm{~mm}$ in diameter and $\sim 45 \mathrm{~mm}$ in length) at a pressure of $250 \mathrm{MPa}$ using a DAKE 44-250 Hydraulic Press at room temperature. Some of these green

Table 1 The mechanical alloying (MA) parameters

\begin{tabular}{lccccc}
\hline & $\begin{array}{c}\text { Milling } \\
\text { materials }\end{array}$ & $\begin{array}{c}\text { Ball-to- } \\
\text { powder } \\
\text { ratio }\end{array}$ & $\begin{array}{c}\text { Rotation } \\
\text { speed, } \\
\text { rpm }\end{array}$ & $\begin{array}{c}\text { Milling } \\
\text { time, h }\end{array}$ & $\begin{array}{c}\text { Milling } \\
\text { atmosphere }\end{array}$ \\
\hline $\begin{array}{l}\text { Planetary } \\
\text { ball mill }\end{array}$ & Steel balls & $10: 1$ & 100 & 16 & Argon (Ar) \\
\hline
\end{tabular}

compacts were then conventional sintered (CS) at $1200{ }^{\circ} \mathrm{C}$ for $6 \mathrm{~h}$ in the tube furnace that was filled with an Ar gas. The other part of the green compacts was then hot isostatic pressed (HIP) in a MiniHIPPer system with a diameter $90 \mathrm{~mm}$ and height $120 \mathrm{~mm}$ hot zone (ABB Autoclave Systems, Inc., Columbus, OH, USA). During the HIP process, the pressure and temperature were increased simultaneously up to $160 \mathrm{MPa}$ and $1200{ }^{\circ} \mathrm{C}$, respectively, from ambient for $6 \mathrm{~h}$. The specimens (HIPed) were then furnace cooled down to room temperature. Part of the HIPed specimens was finally solution-treated at $1000{ }^{\circ} \mathrm{C}$ for $60 \mathrm{~min}$ in $\mathrm{Ar}$ atmosphere followed by water quenching. Therefore, three different porous TiNbZr specimens were examined in this study they were (i) CS-TiNbZr, (ii) HIP-TiNbZr, and (iii) HIP-sstTiNbZr. The densities of all specimens were determined by Archimedes' principle. An Olympus BH2-UMA Optical Microscope (OM) was used to characterize the pore characteristic of the specimens. The HIP-sst-TiNbZr specimen was etched in a solution of $\mathrm{HNO}_{3}(5$ vol. $\%)+\mathrm{HF}(10$ vol. $\%)+$ $\mathrm{H}_{2} \mathrm{O}$ (Bal.) to reveal the martensite using a JEOL JSM-820 Scanning Electron Microscope (SEM). The crystal structures were identified using an X'pert MPD Pro X-ray diffraction instrument with $\mathrm{Cu}-\mathrm{K} \alpha$ target $(\lambda=1.5406 \AA)$ in $2 \theta$ ranges between $30^{\circ}$ and $80^{\circ}$. Cylindrical samples with $3 \mathrm{~mm}$ in diameter and $6 \mathrm{~mm}$ in length were cut to characterize the mechanical properties. Compression tests were performed at room temperature using an Instron 4206 materials test system. The strain rate is $3.33 \times 10^{-3} / \mathrm{s}$.

\section{Results and Discussion}

Figure 1 shows typical optical micrographs of the cross sections of porous TiNbZr alloys fabricated by (a) conventional sintering (CS), (b) hot isostatic pressing (HIP), and (c) hot isostatic pressing followed by solid solution treatment (HIP-sst) with different porosity ratios of $\sim 33.2, \sim 12.1$, and $\sim 12.3 \%$, respectively.

It is obvious that the morphologies and pore distributions between the HIP-TiNbZr and the CS-TiNbZr are completely different. Nearly spherical shape pores, ranging from $\sim 5$ to $30 \mu \mathrm{m}$ in size, distributed quite uniformly on the cross section of the HIP-TiNbZr can be observed in Fig. 1(b), (c). In contrast, mostly irregular and tunnel-like pores, ranging from $\sim 10$ to $50 \mu \mathrm{m}$ in size were found in the CS-TiNbZr (Fig. 1a), indicating that the high pressure imposed during the HIP process plays an important role in determining the pore characteristics. Also, the formation of the relatively spherical pores in the HIPed specimen can be associated with expanding of the gas out-gassed from the powders which was trapped in the specimen during the HIP process. This is in agreement with the findings of Yuan et al. (Ref 1) who investigated the superelastic properties of porous TiNi shape memory alloys prepared by hot isostatic pressing. However, the pore distributions and sizes of both HIP-TiNbZr and HIP-sst-TiNbZr showed nearly the same, suggesting insufficient time and/or temperature to enlarge the pore sizes during further solid solution treatment.

Figure 2 shows the $x$-ray diffraction patterns of (a) the powders after mechanical alloying, (b) HIP-TiNbZr, and (c) HIP-sst-TiNbZr. In Fig. 2(a), it reveals that no alloying had occurred after the ball-milling process. 

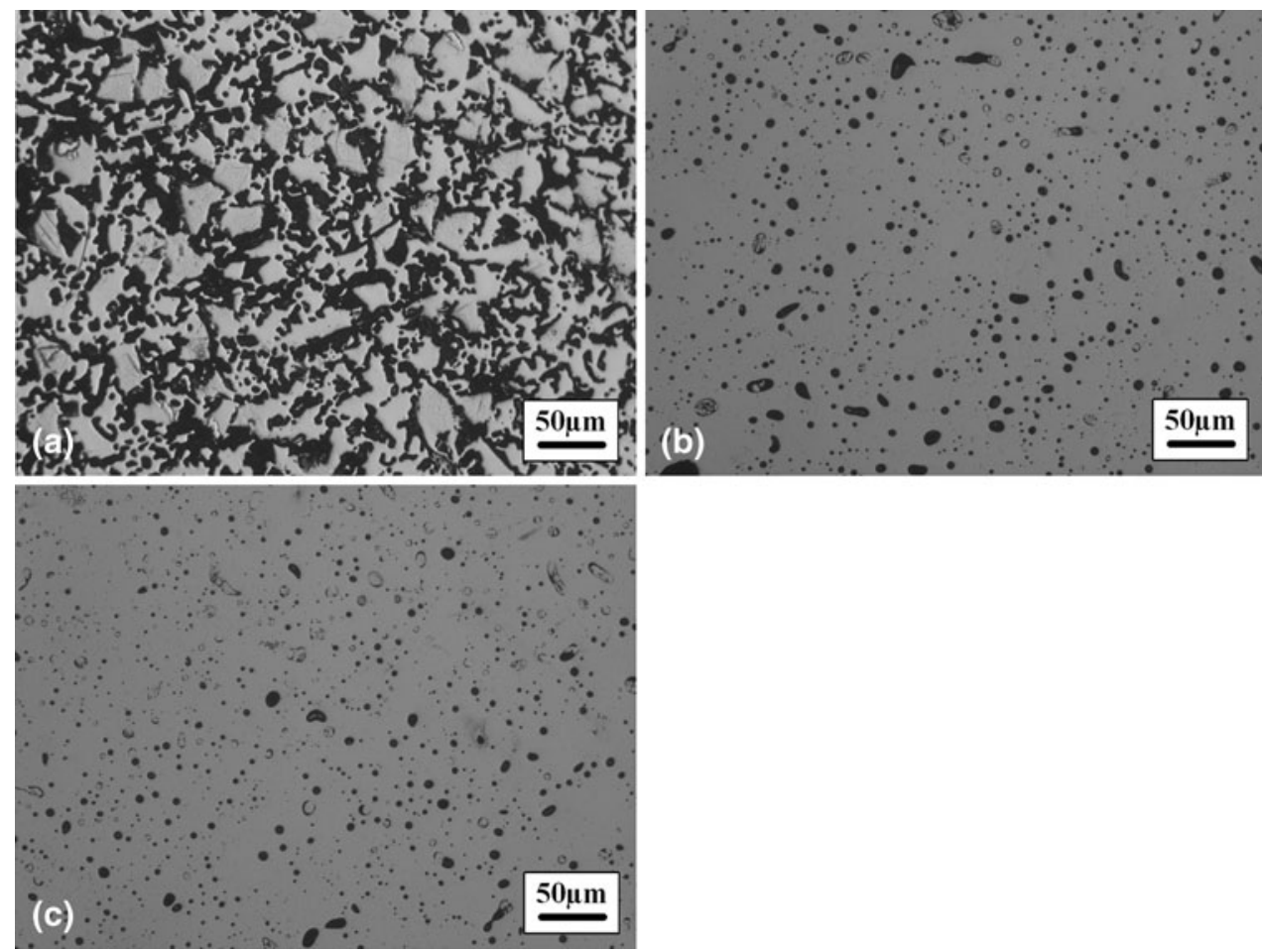

Fig. 1 Typical optical micrographs of the cross sections of porous TiNbZr alloys fabricated by (a) conventional sintering (CS), (b) hot isostatic pressing (HIP), and (c) hot isostatic pressing followed by solid solution treatment (HIP-sst) with different porosity ratios of $\sim 33.2$, $\sim 12.1$, and $\sim 12.3 \%$, respectively

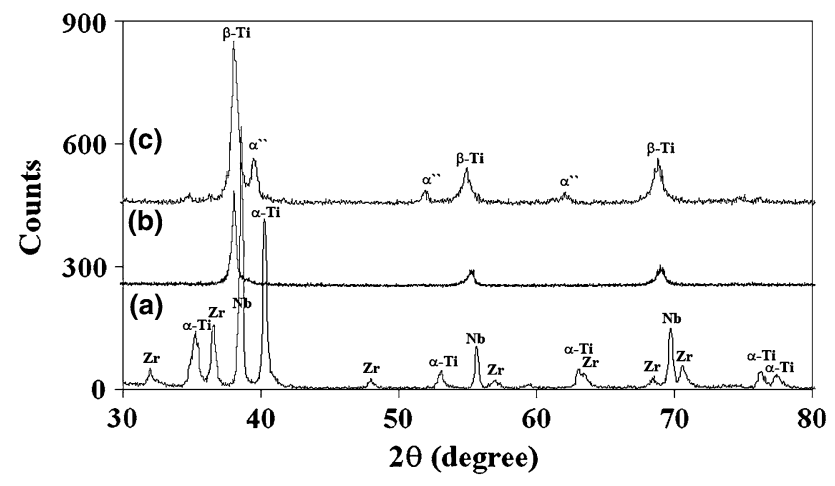

Fig. 2 X-ray diffraction patterns of (a) the powders after mechanical alloying, (b) HIP-TiNbZr, and (c) HIP-sst-TiNbZr

After HIP, there exist only the peaks corresponding to the $\beta$-Ti phase without $\alpha$ phase in the alloy (Fig. 2b). This is in agreement with Kim et al. (Ref 4), who investigated the shape memory characteristics of Ti-22Nb-(2-8)Zr biomedical alloys were fabricated by an arc melting method. They also reported that the martensitic transformation start temperature $\left(M_{\mathrm{s}}\right)$ of the alloys with percentage of $4 \mathrm{Zr}, 6 \mathrm{Zr}$, and $8 \mathrm{Zr}$ were below room temperature due to the absence of $\alpha^{\prime \prime}$ martensitic phase in the XRD profiles. Surprisingly, several peaks corresponding to the coexistence of the $\alpha^{\prime \prime}$ martensitic and the $\beta$-Ti phases were observed in the HIPed specimen after solid solution treatment at $1000{ }^{\circ} \mathrm{C}$ for $60 \mathrm{~min}$ (Fig. 2c). In the present investigation, the peaks corresponding to the $\alpha^{\prime \prime}$ martensitic phase were observed in the HIP-sst-TiNbZr

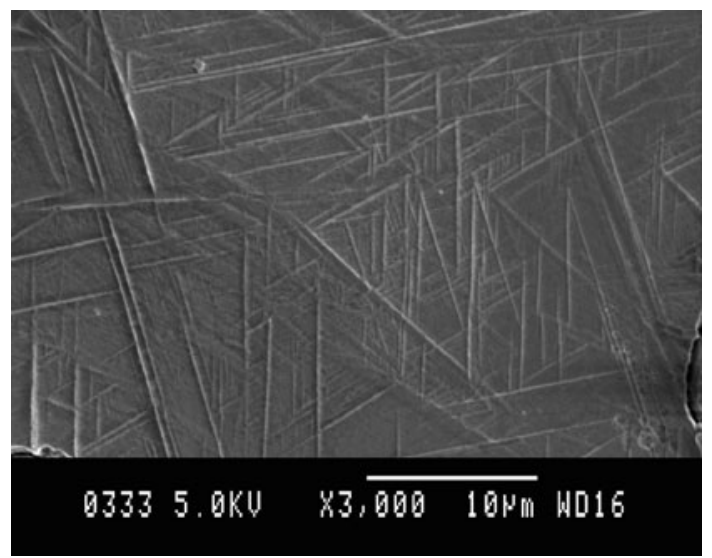

Fig. 3 Secondary electron image of the morphology of martensites

suggesting that the $M_{\mathrm{s}}$ temperature of the alloy is above room temperature. In addition, this is consistent with a secondary electron image of the morphology of martensites shown in Fig. 3. Very clear fine needle-like traces of martensite can be observed.

Previous investigation of Ti-22Nb-6Zr (at.\%) alloys fabricated by arc melting method has shown that these alloys annealed at 773 and $823 \mathrm{~K}$ exhibited peaks corresponding to the $\alpha$ phase, Those annealed above $873 \mathrm{~K}$ exhibited only peaks corresponding to the $\beta$ phase ( $\operatorname{Ref} 15)$. It is thought that deformation texture or re-crystallization texture was obtained to form these phases in the as-rolled or annealed alloys after severe cold rolling. In contrast, no severe deformation is 


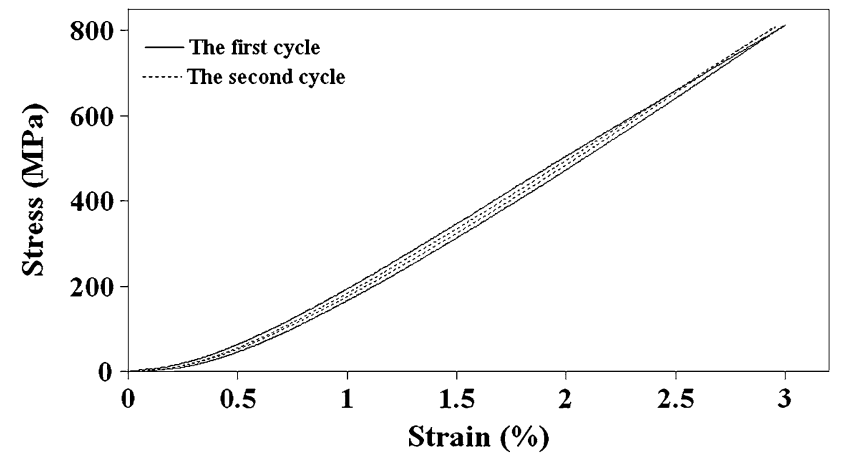

Fig. 4 Compressive stress-strain curve of the HIP-sst-TiNbZr

subjected in the present alloys after either CS or HIP as well as HIP-sst.

Figure 4 shows the compressive stress-strain curve of the HIP-sst-TiNbZr. The test was performed at room temperature. This figure reveals that the HIP-sst-TiNbZr shows nearly complete superelasticity in the first cycle.

The plastic strain was only about $0.1 \%$ after unloading. The HIP-sst-TiNbZr then showed complete superelasticity in the subsequent second compression cycle and its maximum recoverable elastic compressive strain was $\sim 3 \%$. Moreover, it can be noted that the compressive strength of the HIP-sstTiNbZr alloy is up to $\sim 800 \mathrm{MPa}$, which is much higher than the porous NiTi SMA $(\sim 310 \mathrm{MPa})$ with the pores size in a range of $50-300 \mu \mathrm{m}$, studied by $\mathrm{Li}$ et al. (Ref 2) and the compact human bone. It is suggested that the present HIPsst-TiNbZr alloy is able to meet the strength demand of hard tissue implant.

\section{Conclusions}

Ti-22Nb-6Zr (at.\%) shape memory alloy was successfully fabricated using both MA together with HIP methods. Nearly spherical pores distributed on the cross section of the HIPTiNbZr alloy were obtained. Martensitic phases were found in the HIP-sst-TiNbZr alloy and further confirmed by its lamellar morphologies. Moreover, nearly complete superelasticity and $\sim 3 \%$ recoverable elastic compressive strain was achieved after mechanical cycling.

\section{Acknowledgments}

The study described in this article was fully supported by a Grant from the Research Grant Office of the City University of Hong Kong, China (CityU SRG project \#7002215).

\section{References}

1. B. Yuan, C.Y. Chung, P. Huang, and M. Zhu, Superelastic Properties of Porous TiNi Shape Memory Alloys Prepared by Hot Isostatic Pressing, Mater. Sci. Eng. A, 2006, 438-440, p 657-660

2. H. Li, B. Yuan, Y. Gao, M. Zhu, and C. Y. Chung, Properties of Porous NiTi SMAs Fabricated by Low Pressure Sintering, Proceedings of the International Conference on SMST, Tsukuba, Japan, 2007, p 109-114

3. T. Maeshima and M. Nishida, Shape Memory and Mechanical Properties of Biomedical Ti-Sc-Mo Alloys, Mater. Trans., 2004, 45(4), p 1101-1105

4. J.I. Kim, H.Y. Kim, T. Inamura, H. Hosoda, and S. Miyazaki, Shape Memory Characteristics of Ti-22Nb-(2-8)Zr(at.\%) Biomedical Alloys, Mater. Sci. Eng. A, 2005, 403, p 334-339

5. C. Baker, The Shape-Memory Effect in a Titanium-35 wt.-\% Niobium Alloy, Met. Sci. J., 1970, 5, p 92-100

6. H.Y. Kim, H. Satoru, J.I. Kim, H. Hosoda, and S. Miyazaki, Mechanical Properties and Shape Memory Behavior of Ti-Nb Alloys, Mater. Trans., 2004, 45(7), p 2443-2448

7. D.H. Ping, Y. Mitarai, and F.X. Yin, Microstructure and Shape Memory Behavior of a Ti-30Nb-3Pd Alloy, Scripta Mater., 2005, 52, p 12871291

8. S.M. Green, D.M. Grant, and N.R. Kelly, Powder Metallurgical Processing of Ni-Ti Shape Memory Alloy, Powder Metall., 1997, 40(1), p 43-47

9. B. Bertheville, Porous Single-Phase NiTi Processed Under $\mathrm{Ca}$ Reducing Vapor for Use as a Bone Graft Substitute, Biomaterials, 2006, 27, p 1246-1250

10. C.L. Chu, J.C.Y. Chung, and P.K. Chu, Effects of Heat Treatment on Characteristics of Porous Ni-rich NiTiSMA Prepared by SHS Technique, Trans. Nonferrous Met. Soc. China, 2006, 16, p 49-53

11. Y. Zhao, M. Taya, Y.S. Kang, and A. Kawasaki, Compression Behavior of Porous NiTi Shape Memory Alloy, Acta Mater., 2005, 53, p 337343

12. A. Bansiddhi and D. Dunand, Shape-Memory NiTi Foams Produced by Solid-State Replication with NaF, Intermetallics, 2007, 15, p 16121622

13. Y.P. Zhang, B. Yuan, M.Q. Zeng, C.Y. Chung, and X.P. Zhang, High Porosity and Large Pore Size Shape Memory Alloys Fabricated by Using Poreforming Agent $\left(\mathrm{NH}_{4} \mathrm{HCO}_{3}\right)$ and Capsule-Free Hot Isostatic Pressing, J. Mater. Process. Technol., 2007, 192, p 439-442

14. C.Y. Chung and L.W. Ma, NiAl Shape Memory Alloy Prepared by Hot Isostatic Pressing, J. Phys. IV, 2003, 112, p 1059-1062

15. J.I. Kim, H.Y. Kim, T. Inamura, H. Hosoda, and S. Miyazaki, Effect of Annealing Temperature on Microstructure and Shape Memory Characteristics of Ti-22Nb-6Zr(at.\%) Biomedical Alloy, Mater. Trans., 2006, 47(3), p 505-512 